Sir,

\section{Reply to Dr Haigis}

Dr Haigis' comment about the nomenclature is very justified. About $\mathrm{ACD}=\mathrm{AtoACD}(\mathrm{ELPtoA}($ ELPconst)), this function converts ELP constant to ACD constant for the particular IOL. As can be seen in the definition of the HofferQ function in the appendix, ACD is first defined as a variable of the double type and is assigned the value of ACD constant (personalised ACD constant or manufacturer's ACD constant, whatever) in the first step; then this value is modified as we go down the next steps inside the function. Unfortunately, the whole subject of biometry is replete with overlapping and sometimes confusing terms, for example, ACD (is it ac depth or ELP?), corneal height (from corneal vertex or from secondary principal plane or posterior surface?), $K$ value (optical or keratometric?), axial length (ultrasonic or optical?), etc.

About the computation of the predicted ACD according to Dr Hoffer's 1993 paper, in my article I did mention that I already experimented with all these data and it gave identical values of the predicted ACD $(4.40887,3.14482$, and 6.06225 in examples 1,2 , and 3, respectively). I think our confusion is from the supposition of ELP constant being equal to ACD constant, which it is not.

\section{S Basu}

Department of Ophthalmology, Apollo Gleneagles Hospital, 58, Canal Circular Road, Kolkata 700054, India

Correspondence: S Basu,

Tel: + 91332320 3040;

Fax: + 913323205218.

E-mail: sbasu2003@doctors.net.uk

Eye (2007) 21, 550. doi:10.1038/sj.eye.6702560; published online 29 September 2006

Please note that this article is linked to the following Correspondence articles, which were published in the March 2007 issue of Eye (issue 21.3):

Hoffer KJ. Errors in self-programming the Hoffer Q formula. Eye 2007; 21: 429. doi:10.1038/sj.eye.6702559.

Basu S. Reply to Dr Hoffer. Eye 2007; 21: 430. doi:10.1038/ sj.eye.6702561.

Haigis W. Reply to Basu S et al. Eye 2007; 21: 430-431. doi:10.1038/sj.eye.6702562.
Sir,

Reply to letter from Cooke et al: silicone oil migration causing increasing proptosis $\mathbf{1 3}$ years after retinal surgery

This letter purports to attribute the late complications of unsuccessful surgery for the repair of retinal detachment due to trauma, to silicone oil. The eye in question is certainly unsightly, but there is no evidence from the report that the condition was progressive as implied in the title. The eye was said to have been more prominent only for 6 months, not progressively so. The title implies that the proptosis was due to the silicone; yet the CT scan shows a mass to the medial side of the eye, with limited spread more posteriorly. Had the proptosis been related to the extraocular movement to the silicone, there would have been evidence of a mass behind the eye.

The eye was reported as showing axial myopia as evidenced by an A-scan measurement. The authors should surely have known that ultrasound measurements in the presence of silicone oil always show an abnormal axial length owing to attenuation of the sound signal through the silicone. However, the CT scan does show an enlarged eye with some lateral displacement owing to a medially situated mass. It is highly probable that this previously traumatised eye had shown progressive enlargement due to glaucoma complicating unsuccessfully treated retinal detachment as evidenced by the appearance of the cornea. This would have led to a high risk of exposure and an unsightly eye. It is noteworthy that there appeared to be no history of pain from this eye.

It is also noteworthy that the histopathology showed only a relatively mild inflammatory reaction. This therefore could not be regarded as a granuloma. This term should be restricted to a mass involving chronic inflammation.

Silicone oil may leak from a glaucomatous eye, such as the one described in this letter. It is the result of scleral rupture, usually at one of the parsplanar sclerostomies used for vitrectomy. We have seen this in some patients where oil droplets leak slowly beneath the conjunctiva. It does not however cause such a red and unsightly eye unless other problems are present. In this case, it is highly likely to have been the result of corneal exposure and not oil 'granuloma'.

The analogy with historic techniques for breast augmentation has been used by critics of the use of silicone oil for the treatment of complex retinal detachment. The comment in this letter begins with the statement that oil granuloma occurs when bulky mineral oils are injected into body tissues. It is true that serious problems did occur with the use of mineral oil for breast 
augmentation and was rapidly discontinued as a result. It is however highly misleading to use this analogy in any discussion regarding silicone oil. Silicone is not a mineral oil; it is not derived from petroleum and is never likely to be.

MP Snead and JD Scott

Addenbrooke's Hospital, Vitreoretinal Service,

Cambridge, Cambridgeshire CB2 2QQ, UK

Correspondence: MP Snead,

Tel: + 441223216701 ;

Fax: + 441223217968.

E-mail: mps34@cam.ac.uk

Eye (2007) 21, 550-551. doi:10.1038/sj.eye.6702631

published online 24 November 2006

$\operatorname{Sir}$

Reply to 'Silicone oil migration causing increasing proptosis 13 years after retinal surgery'

We thank Snead et al for their comments in relation to our recent paper, which enable us to explain elements of the report, which may require further clarification.

The indication we have that this condition was progressive was from the patient himself. He had failed to attend for follow-up appointments following his original surgery. However, he stated that his eye had started to become more prominent approximately 6 months before he reattended despite relative stability in the preceding decade.

The CT scan shows enhancement of the soft tissues, mainly around the medial rectus, but there are changes extending behind the globe medially. This mass in itself would not cause an axial proptosis, rather a non-axial proptosis. We agree that the CT scan shows an enlarged right eye and axial length measurements were specifically obtained as this could have been considered a compounding factor in the apparent prominence of the right eye. However, the relatively rapid change over 6 months reported by the patient suggests that factors other than axial elongation were involved. We are aware that A-scan ultrasound is attenuated in the presence of silicone oil owing to the lower sound velocity. The sound velocity in silicone oil depends on the viscosity of the oil used. In this case, the oil was 1000 centistokes and therefore the velocity used was $980 \mathrm{~min} / \mathrm{s}$ and this was taken into account when performing the applanation A-scan biometry. Notwithstanding the accuracy of A-scanning in silicone oil, the authors agree that the CT scan does show an enlarged eye.
Although the patient did not report pain from the eye at any stage, we agree that the corneal changes are consistent with old decompensation. Whether this was due to exposure or to complete corneal decompensation as a result of the initial trauma, surgery, or silicone oil contact with the corneal endothelium, it is difficult to determine. It is likely that all played a part. We have no way of determining whether he had developed a secondary glaucoma, as he had not attended for any follow-ups. However, it is highly likely that at some stage he developed glaucoma as this is a known complication of silicone oil. However, as regards the possibility of (relatively rapid) enlargement of the globe from glaucoma, we feel this is unlikely. Certainly, if glaucoma develops in a child the globe will enlarge as a result of the elasticity of the scleral and corneal tissue. The cornea can enlarge up until about age 3 years, but the sclera can continue to deform until about age 10 years. ${ }^{1}$ In an adult with normal sclera, this scenario typically does not occur as the adult globe is no longer distensible because of crosslinking of the scleral collagens. The eye wall can stretch with raised intraocular pressure but scleral stretch is minimal and totally reversible without permanent globe enlargement. ${ }^{2}$

We confirm that at the time of enucleation there was clear evidence of large glistening globules of silicone oil throughout the peri- and retro-orbital tissues particularly medially and the conjunctival and tenons layers were extremely adherent together. Therefore, clinically there was no doubt that silicone oil had been actively leaking around the globe. We described the inflammation around the silicone as 'mild inflammation'. We did not describe the inflammation as granulomatous, as we agree it is not 'granulomatous inflammation' in the strict histopathological use of the term. However, the term 'granuloma' is used by pathologists to describe any small nodular delimited aggregation of mononuclear cells and the definition of granuloma is therefore appropriately used when describing 'oil granuloma' and 'silicone granuloma'.

We had also not previously seen such an inflamed eye from silicone oil leakage before and felt that this case was worth reporting, as it was an unusual occurrence. We believe that 'corneal exposure' alone cannot account for the widespread changes reported. The vascularised corneal changes were long-standing and there was no associated epithelial defect or evidence of exposure. The large gelatinous subconjunctival mass seen in the clinical photograph was highly unusual in appearanceprompting the biopsy. Our initial clinical diagnosis included lymphoma but the biopsies were negative for this demonstrating the silicone oil changes only.

In our paper, we make the statement 'Oil granuloma occurs when bulky mineral oils are injected into body tissues'. One widely accepted definition of 'mineral' is any inorganic substance, and of 'oil' is a greasy liquid. By 